\title{
Microwave brightness temperature imaging and dielectric properties of lunar soil
}

\author{
Wu Ji ${ }^{1, *}$, Li Dihui ${ }^{1}, Z_{h a n g}$ Xiaohui ${ }^{1}$, Jiang Jingshan ${ }^{1}$, A T Altyntsev ${ }^{2}$ \\ and B I LUBYSHEV ${ }^{2}$ \\ ${ }^{1}$ Center for Space Science and Applied Research, Chinese Academy of Sciences, \\ P.O. Box 8701, Beijing 100 080, China. \\ ${ }^{2}$ Institute of Solar Terrestrial Physics, Siberia Branch of Russian Academy of Sciences, \\ P.O. Box 4026, Irkutsk 664 033, Russia. \\ ${ }^{1,2}$ China-Russia Joint Research Center on Space Weather \\ *e-mail:wuji@center.cssar.ac.cn
}

\begin{abstract}
Among many scientific objectives of lunar exploration, investigations on lunar soil become attractive due to the existence of $\mathrm{He}^{3}$ and ilmenite in the lunar soil and their possible utilization as nuclear fuel for power generation. Although the composition of the lunar surface soil can be determined by optical and $\gamma / \mathrm{X}$-ray spectrometers, etc., the evaluation of the total reserves of $\mathrm{He}^{3}$ and ilmenite within the regolith and in the lunar interior are still not available. In this paper, we give a rough analysis of the microwave brightness temperature images of the lunar disc observed using the NRAO 12 meter Telescope and Siberian Solar Radio Telescope. We also present the results of the microwave dielectric properties of terrestrial analogues of lunar soil and, discuss some basic relations between the microwave brightness temperature and lunar soil properties.
\end{abstract}

\section{Introduction}

China plans to launch a lunar exploration orbiter, Chang'E-1, in the coming few years. A microwave radiometer will be on this mission to collect lunar brightness temperature data for determining the thickness of the lunar soil and other soil characteristics.

The Moon is the only and the nearest natural celestial body revolving around the Earth. In view of its uniqueness, it was the first object of study and has been very attractive since ancient times. Study of the lunar microwave brightness temperature began in the 1940s, represented by the work of Dicke and Beringer (1946) and Piddington and Minnett (1949). They discovered the approximate sinusoidal change of lunar brightness temperature with the lunar phase. In the 1950s, Troitskii made further study of lunar brightness temperature and quantified some of the lunar radio emission properties and regularities discovered by earlier researchers (Hagfors et al 1969). With the establishment of the large-scale radio astronomical telescope in the 1960s and early 1970s, many scientists joined the study of lunar brightness temperature. The wavelengths used in observation ranged from $168 \mathrm{~cm}$ to $1 \mathrm{~mm}$, covering nearly all the microwave frequencies. But these studies stopped when Neil Armstrong set his foot on the lunar surface at 02:56:15 UT on 21st July 1969. From then on, a great number of scientists from different disciplines were involved in the study of lunar material collected by former Soviet Union robots and Apollo astronauts. With the completion of the first round of lunar exploration by human beings, the study of lunar microwave brightness temperature was completely forgotten. Accompanied by a new upcoming era of lunar exploration and the development of science and technology, observation of the lunar microwave brightness temperature

Keywords. Microwave brightness temperature; dielectric constants of lunar soil; microwave radiometer. 


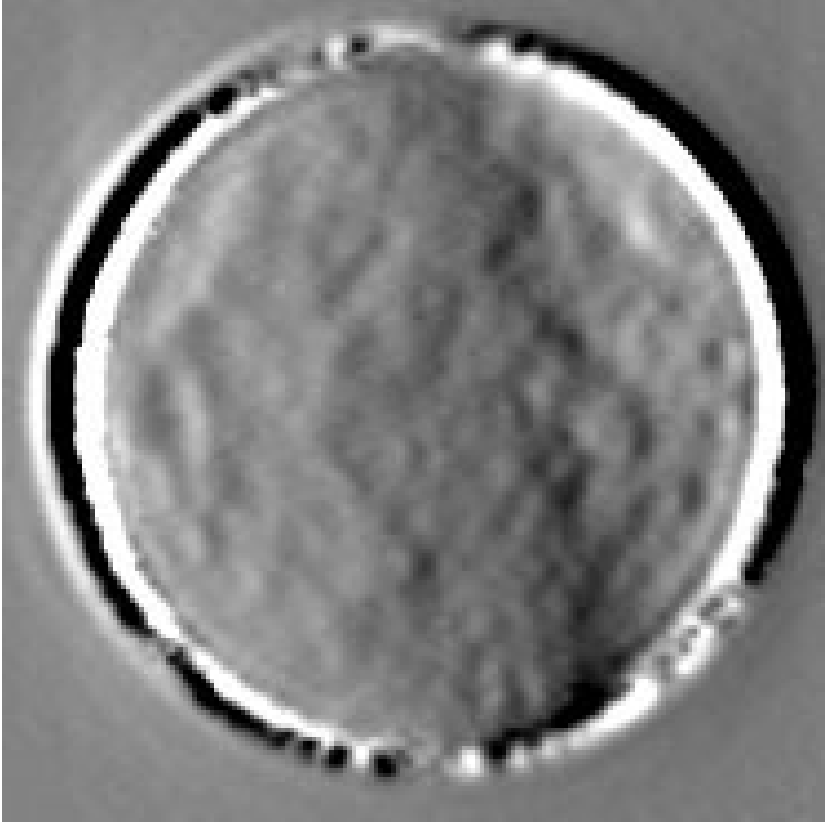

Figure 1. Microwave brightness temperature image of the Moon at $90 \mathrm{GHz}$.

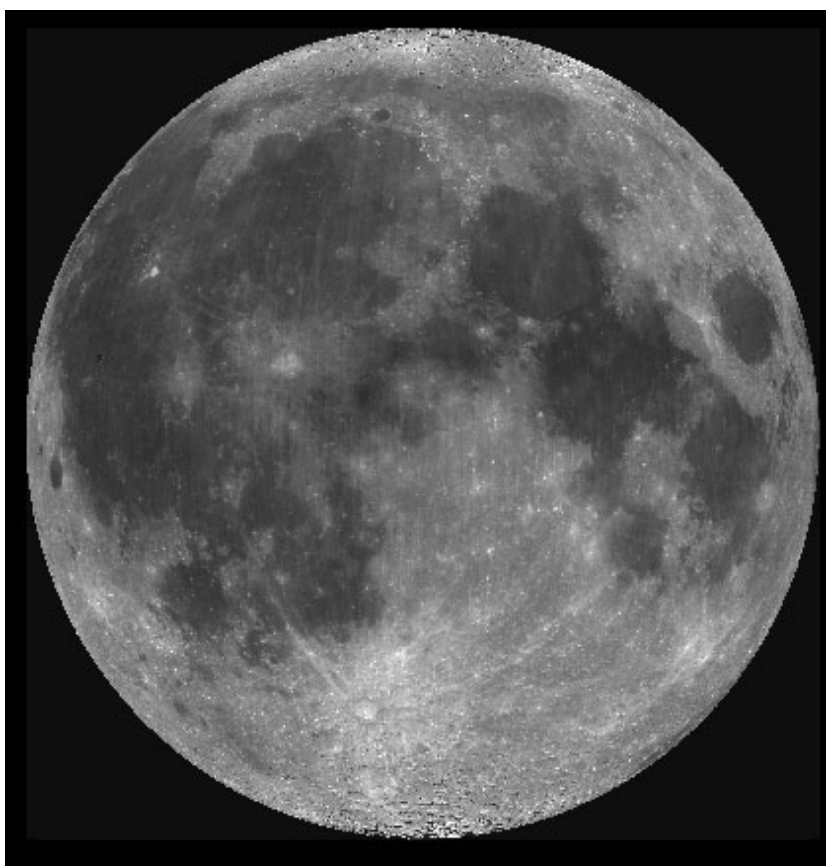

Figure 2. Optical image of the Moon.

has again become important for exploration of water-ice, characterizing thickness of lunar soil and lunar resources. In this paper, we first explain two significant lunar microwave brightness temperature images observed with the ground-based radio astronomical telescopes in recent years and then describe our study of the microwave dielectric properties of lunar soil, and finally, discuss the possible relations between microwave brightness temperature and depth of the lunar soil.

\section{Lunar microwave brightness temperature}

For a long time, visible light and infrared have been the preferred wavelengths for lunar observations though microwave band was also used occasionally. Because of the synchronization of the Moon's rotation and revolution, visible and infrared light cannot 'see' the dark side of the Moon, but microwave can. As we know, it has the 'ability' to 'see' things in the dark and there is no need for solar illumination. Moreover, it can penetrate a certain depth under the surface of dielectric material. Therefore, microwaves can be used for the Moon's all-time observation, and study of the Moon's microwave brightness temperature can probably be useful in obtaining information of the depth and structures of the lunar soil. Figure 1 shows the microwave brightness temperature image of the Moon's surface observed on April 1st, 1994 using the NRAO 12 meter Telescope at Kitt Peak, Arizona at a frequency of $90 \mathrm{GHz}(3.3 \mathrm{~mm})$. Figure 2 is its nearly corresponding optical black and white image. The radio surface of figure 1 appears different from the familiar optical surface shown in figure 2. In comparison to the optical image (figure 2), the microwave image of the Moon (figure 1) roughly reflects the topographic characteristics of mare and highlands, the protrusion extending $\mathrm{S}-\mathrm{N}$ in the middle of the figure nearly corresponds to the lunar highland terrain. Figure 3 is the microwave brightness temperature image of the lunar disc with 20 arc-sec angular resolution observed on October 27th, 2001 using the Siberian Solar Radio Telescope (SSRT). The lunar phase was nearly $70 \%$ during the observation period. Different from figure 1 , the brighter region in figure 3 is approximately in accordance with the mare area in the upper right of the optical moon shown in figure 2. We expected that figure 1 would show the temperature features of the lunar surface and that figure 3 would reflect the properties up to a certain depth under the surface.

\section{Dielectric properties of the lunar soil simulators}

The microwave dielectric constant is an important parameter for interpretation of the lunar brightness temperature images. In order to understand the microwave brightness temperature data expected from Chang'E's microwave radiometer, nine terrestrial basalts and anorthosites were selected based on the similarity of their chemical compositions to various lunar soils, as determined by X-ray fluorescence. We measured the relative dielectric constants of these lunar analogues at five densities $\left(0.8,1.0,1.2,1.4,1.6 \mathrm{~g} / \mathrm{cm}^{3}\right)$ over the range of 


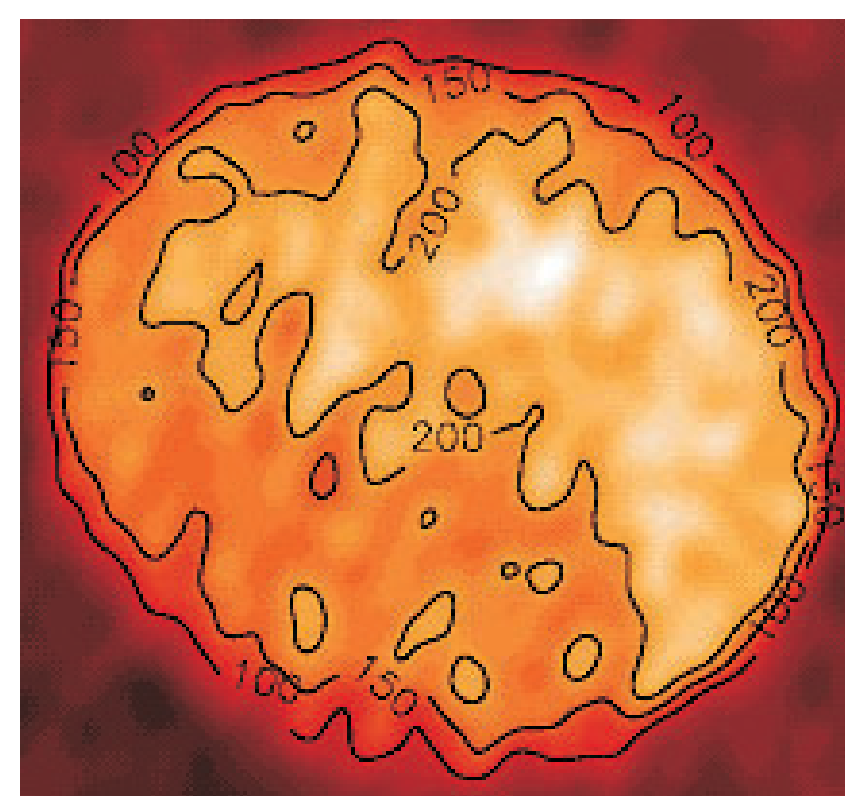

Figure 3. Microwave brightness temperature image of the Moon at $5.7 \mathrm{GHz}$.

0.5-20 GHz with open-ended coaxial line model on the HP8722C Network Analyzer. The measurement accuracy of the model is \pm 0.05 for both real part $\varepsilon^{\prime}$ and loss tangent $\varepsilon^{\prime} / \varepsilon^{\prime \prime}$, and the error caused by measurement operation is generally \pm 0.02 . All the measurements are given in an experiment report ( $\mathrm{Li}$ Dihui et al 2004) and each of them is the average of 3 to 5 sets of measurement values in different positions. Changes of the real parts $\varepsilon^{\prime}$ and imaginary parts $\varepsilon^{\prime \prime}$ of the relative complex dielectric constants with density and frequency are shown in figures 4 and 5, where different curves represent the samples with different contents of $\mathrm{Ti}$ and Fe oxides.

\subsection{Quantitative characteristics of the relative complex dielectric constants}

Variance tests and polynomial regression indicate that density, frequency and composition contribute to the relative complex dielectric constants. Density is the most dominating factor followed by frequency whereas composition shows a complicated situation. Averages of the real parts of the relative complex dielectric constants at five densities $0.8,1.0,1.2,1.4$ and $1.6 \mathrm{~g} / \mathrm{cm}^{3}$ are 2.1560 , $2.4425,2.7098,3.0729$ and 3.4288 respectively and those of the imaginary parts are $0.0844,0.1024$, $0.1208,0.1627$ and 0.1922 respectively. The maximum of the real parts is approximately 1.6 times the minimum and the maximum of the imaginary parts is about 2.3 times the minimum. In the range of frequency from $500 \mathrm{MHz}$ to $20 \mathrm{GHz}$, the maximum difference between the real parts is 0.8816 and that between the imaginary parts is 0.738 .

\subsection{Relations between relative complex dielectric constant and density}

Real parts and imaginary parts of the relative complex dielectric constants are strongly affected by the sample density. Figure 4 shows the projections of the relative dielectric constants of different analogues vs. density at different microwave frequencies on a planar rectangular coordinate system. Different curves represent terrestrial analogues of lunar samples having different $\mathrm{Fe}$ and Ti concentrations (for both, real and imaginary parts). It shows that the real parts have a quicker increase with increased density at the same frequency and all the samples fall in a narrow belt. Regression analyses indicate that a linear relation exists between the real parts and the density over the range of $0.5-20 \mathrm{GHz}$ and the correlation coefficients average 0.989 . The imaginary parts show a similarity to the real parts over the range of $>0.5-10 \mathrm{GHz}$, but begin to change irregularly at about $10 \mathrm{GHz}$. Accordingly, the imaginary parts under $10 \mathrm{GHz}$ are linearly correlated with the density with the average correlation coefficient of $\sim 0.94$, which drops to 0.69 in the range of all measuring frequencies.

\subsection{Relations between relative complex dielectric constant and frequency}

Figure 5 shows the projections of the relative dielectric constants of different simulators $v s$. microwave frequency at different densities on a planar rectangular coordinate system. Real parts and imaginary parts of the relative complex dielectric constants demonstrate totally opposite changes with frequency. With the increase of microwave frequency, the real parts tend to decline whereas the imaginary parts rise. Regression analyses indicate that real parts are also linearly but negatively correlated with microwave frequency in the range of $500 \mathrm{MHz}$ to $20 \mathrm{GHz}$, and the correlation coefficients average 0.73 , obviously lower than those between the real parts and density. Similar to the changes observed with density, the projection curves of the imaginary parts vs. frequency extend smoothly over the $0.5-10 \mathrm{GHz}$ range, but fluctuate in $10-20 \mathrm{GHz}$, with two to three jumps up and down. The calculations indicate that most of the imaginary parts still vary linearly with increased microwave frequency, but the correlation becomes weaker as indicated by the average correlation coefficient of about 0.34 .

\subsection{Relations between relative complex dielectric constant and composition}

Analysis of measurements reveals that the relative complex dielectric constants and $\mathrm{TiO}_{2}$ or Fe-oxides 

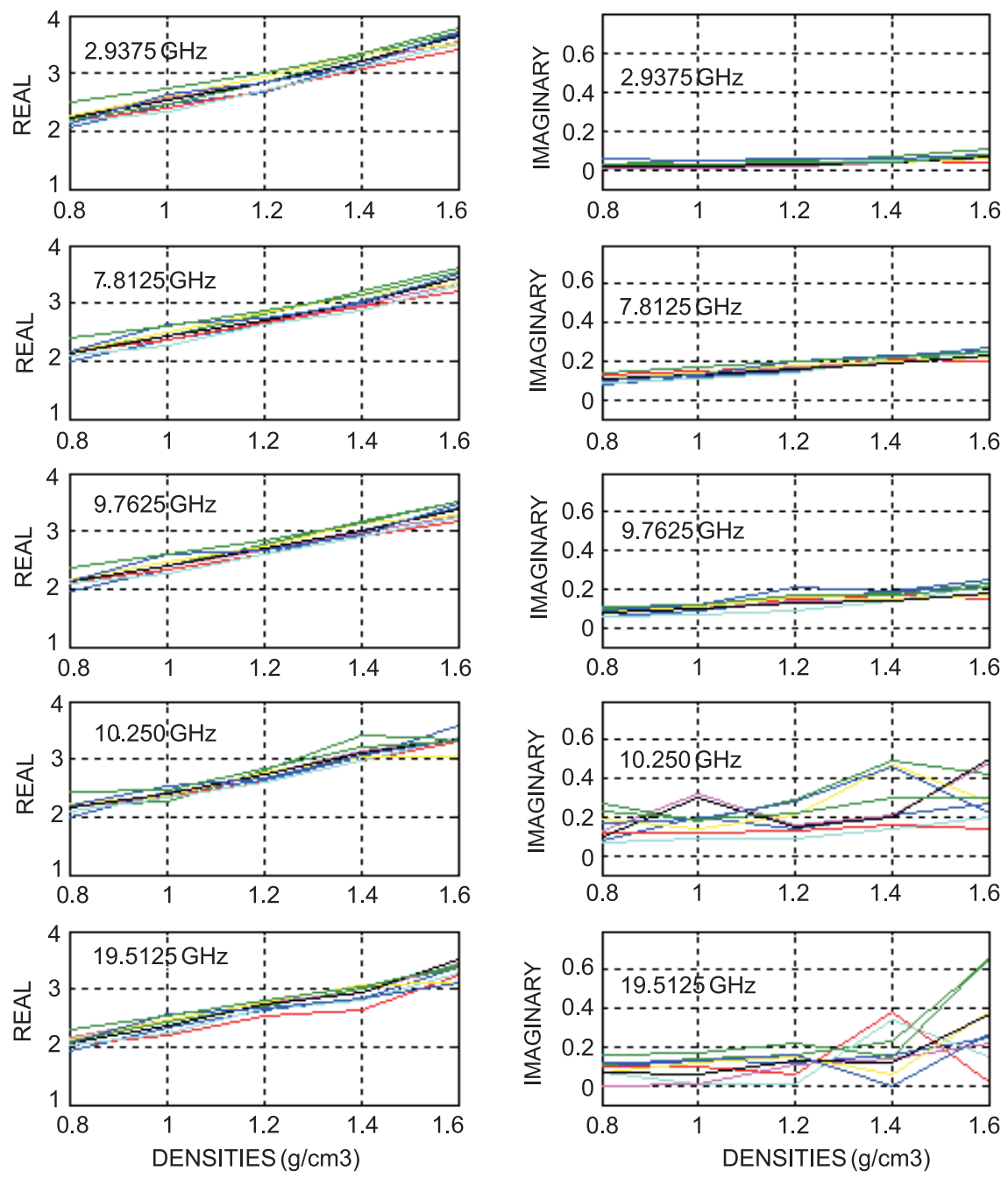

Figure 4. Changes of relative complex dielectric constant (real and imaginary part) with density (the colored lines in the diagrams represent samples with different weight percentages of $\mathrm{TiO}_{2}$ content: blue $-1.0545 \%$, dark green $-1.3589 \%$, red $-2.4877 \%$, cyan $-2.8983 \%$, pink $-3.6799 \%$, light brown $-3.9355 \%$, black $-7.2076 \%$, light blue $-8.2427 \%$, light green $-11.1473 \%$ ).

or $\mathrm{TiO}_{2}+$ Fe-oxides or several main element oxides content do not follow a simple relation and $\mathrm{TiO}_{2}$ or Fe-oxides or $\mathrm{TiO}_{2}+\mathrm{Fe}$-oxides do not contribute to the dielectric constants. The correlation coefficients between $\mathrm{TiO}_{2}$ contents and the relative complex dielectric constants average 0.5 for the real part and 0.24 for the imaginary part. Multiple regressions analysis was made by taking the $\%$ wt. of major element oxides of $\mathrm{SiO}_{2}, \mathrm{Al}_{2} \mathrm{O}_{3}$, $\mathrm{CaO}, \mathrm{MgO}, \mathrm{Na}_{2} \mathrm{O}, \mathrm{TiO}_{2}$ and $\Delta \mathrm{Fe}$ as the arguments of chance variables and each real or imaginary part of the relative dielectric constants as their dependent variable. The results are in accordance with a multivariate linear function. In view of the extremely diversified combinations of material chemical compositions and the entirely different microstructures among the combinations, the effect of the composition on the relative dielectric constants is probably much more complicated than those of density and frequency, and the precise mathematical and physical links between relative dielectric constant and composition need to be studied more.

It is interesting that the dielectric properties of lunar soil analogues are close to those of true lunar samples (Heiken et al 1991), but vary differently from those of terrestrial rocks (Ulaby et al 1990). Thus we believe that the real lunar soil is realistically simulated in dielectric properties to the lunar soil analogues used in this study.

Understanding of the microwave dielectric properties of materials is important for the application of remote sensing techniques. Some workers have confirmed the correlations between the microwave dielectric constants of a material and its microwave brightness temperature (Lai et al 1982; Teng et al 1984; Xiao 1988). For example, they have successfully distinguished between water and land, some distinct types of rocks, different structural formations and iron deposit bodies by 

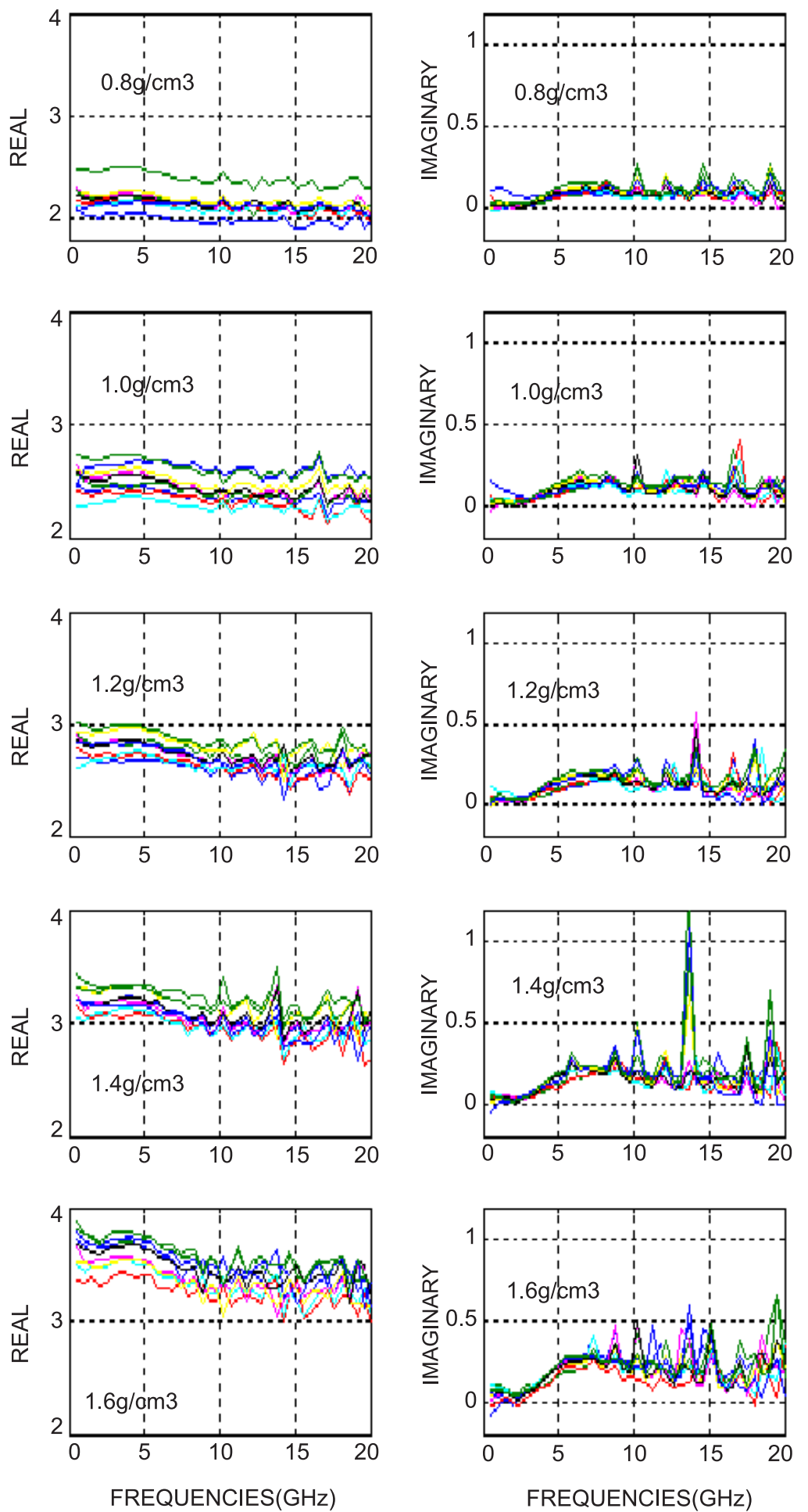

Figure 5. Changes of relative complex dielectric constant with frequency (see figure 4 for the meaning of the colored lines in the diagrams).

means of such correlations. Therefore, it is possible that further careful study of microwave dielectric constants of materials and their corresponding microwave brightness temperature data such as shown in figures 1 and 3 will be helpful in determining the regolith thickness, composition, structure and other physical parameters.

\section{Discussion on microwave brightness temperature and lunar soil}

As we have discussed above, different media have different microwave brightness temperatures, and the microwave brightness temperatures contain information of media density, composition and 
structure, etc. The physical relation of microwave brightness temperature $\left(B_{t}\right)$ and medium is given by

$$
B_{t}=e\left(d, \varepsilon^{\prime}, \varepsilon^{\prime \prime}\right) T
$$

where $T$ is the physical temperature of a medium and can be measured by other means, $e\left(d, \varepsilon^{\prime}, \varepsilon^{\prime \prime}\right)$ is the microwave emissivity of the medium varying between one and zero, a function of $d$ (density), $\varepsilon^{\prime}$ is the real part of the dielectric constant of the medium, $\varepsilon^{\prime \prime}$ the imaginary part of the dielectric constant, where the polarization and viewing angles are assumed to be constant and therefore dependence on these parameters is not discussed here.

A metallic medium is generally considered to be 'cold'. Therefore, it has a low $e$ value, close to 0 , whereas a dense non-conductive dielectric medium is considered 'warm', with a high $e$ value close to 1.0. So, a non-conductive dielectric medium has a high value in $\varepsilon^{\prime}$ and correspondingly shows a high microwave brightness temperature, and a highly conductive medium has high $\varepsilon^{\prime \prime}$ value, with low microwave brightness temperature. In view of its stronger effect on the $\varepsilon^{\prime}$ of a dielectric medium, the density, in fact, plays the role of an amplifier of microwave brightness temperature. The denser the medium, the larger the $e$ value and the higher the microwave brightness temperature. The microwave frequency, however, just shows the opposite effect.

Lunar soil is a non-conductive medium. As inferred from Lai et al (1982) and our dielectric measurements, in an area with similar composition, the lunar soil with a higher brightness temperature should indicate its higher density. In particular, the lunar rocks have higher $\varepsilon^{\prime}$ or high density. Therefore, areas where the regolith layer is not thick or has rocks directly below or is covered with lunar rocks, will appear brighter in a microwave brightness temperature image than the area covered with thicker regolith.

The lower frequency would result in a higher microwave brightness temperature (as shown in figure 5) after absolute calibration. The slope of the measurements from low to high frequency band can also reflect the density of the area. Larger slope reflects higher density as shown in figure 5 and can be used in determining the density.

Limited by the measurement conditions, the mechanism of dependence of composition on dielectric properties of a medium is still not understood. We, however, believe that lunar soil composition (especially with high content of $\mathrm{TiO}_{2}$ or Feoxides) plays an important role in the microwave brightness temperature in view of the rough correspondence between the brighter regions seen in figure 3 and its correspondence with the mare area, in view of the higher dielectric constant values obtained by Chung et al (1971).

\section{Conclusions}

The microwave brightness temperature images of the Moon possibly contain new information about properties of lunar rocks and regolith which are not available in optical images.

Our studies on the microwave dielectric properties of lunar soil analogues and the analysis of the microwave brightness temperature images suggest that denser lunar soil will appear brighter on a microwave brightness temperature image and lower frequency band brightness temperature will correspond to a higher response from lunar soil. These two characteristics will enable us to extract properties of lunar soil with different densities from images in different frequency bands.

\section{References}

Chung D H, Westphal W B and Simmons G 1971 Dielectric behavior of lunar samples: Electromagnetic probing of the lunar interior; Geochim. Cosmochim. Acta Suppl. 2 2381-2390.

Dicke R H and Beringer R 1946 Microwave radiation from the sun and the moon; Astrophys. J. 103(3) 375-376.

Hagfors T, Green J L and Guillen A 1969 Determination of the albedo of the Moon at a wavelength of 6 meter; Astron. J. 74 1214-1219.

Heiken G H, Vaniman D T and French B M 1991 Lunar Sourcebook: a user's guide to the moon; (New York, Port Chester, Melbourne, Sydney: Cambridge University Press) 536-552.

Lai Zhaosheng, Xiao Jinkai and Feng Junming 1982 Dielectric constant determination of some minerals and rocks under microwave frequencies; In: Researches on Mineral Physics and Mineral Materials (Beijing: Science Press) 108-114.

Li Dihui et al 2004 Dielectric properties of several powdered metal-oxide mixtures and lunar soil simulants at varied microwave frequencies. Center for Space Science and Applied Research; Chinese Academy of Sciences, Experimental Report, 1-48 (in Chinese).

Piddington J H and Minnett H C 1949 Microwave thermal radiation from the moon; Aust. J. Sci. Res. A2 63.

Teng Xuyan, Xiao Jinkai, Shi Changging, Lai Zhaosheng, Peng Hongxian and Yang Bolin 1984 Passive microwave radiometry in the Gobi-Desert region; Remote Sensing of Environment 15 37-46.

Ulaby F T et al 1990 Microwave Dielectric Properties of Dry Rocks; IEEE Transactions on Geoscience and Remote Sensing 28(3) 325-336.

Xiao Jinkai 1988 Dielectric property research of minerals and rocks and its significance on remote sensing; Environmental Remote Sensing 3 135-146 (in Chinese). 